\title{
Web-based calculator for biliary atresia screening in neonates and infants with cholestasis
}

\author{
Dongying Zhao ${ }^{1 \#}$, Shengli Gu${ }^{2 \#}$, Xiaohui Gong ${ }^{3 \#}$, Yahui Li ${ }^{1}$, Xiaoang Sun ${ }^{4}$, Yan Chen ${ }^{1}$, Zhaohui Deng ${ }^{4}$, \\ Yongjun Zhang ${ }^{1}$
}

${ }^{1}$ Department of Neonatology, Xinhua Hospital, Shanghai JiaoTong University School of Medicine, Shanghai, China; ${ }^{2}$ Department of Ultrasound, Xinhua Hospital, Shanghai JiaoTong University School of Medicine, Shanghai, China; ${ }^{3}$ Department of Neonatology, Shanghai Children's Hospital, Shanghai Jiao Tong University, Shanghai, China; ${ }^{4}$ Department of Pediatric Digestive Diseases, Shanghai Children's Medical Center, Shanghai Jiao Tong University School of Medicine, Shanghai, China

Contributions: (I) Conception and design: Y Zhang, Y Chen; (II) Administrative support: Y Zhang; (III) Provision of study materials or patients: S Gu, X Gong, Z Deng, Y Zhang; (IV) Collection and assembly of data: D Zhao, S Gu, Y Li, X Sun; (V) Data analysis and interpretation: Y Chen, D Zhao; (VI) Manuscript writing: All authors; (VII) Final approval of manuscript: All authors.

\#These authors contributed equally to this work.

Correspondence to: Yongjun Zhang; MD, PhD. Yan Chen, MD, PhD. Department of Neonatology, Xinhua Hospital, Shanghai JiaoTong University School of Medicine, 1665 Kong Jiang Road, Shanghai 200092, China. Email zhangyongjun@sjtu.edu.cn; chenyan783563@163.com. Zhaohui Deng, PhD. Department of Pediatric Digestive Diseases, Shanghai Children's Medical Center, Shanghai JiaoTong University School of Medicine, 1678 Dongfang Road, Shanghai 200127, China. Email dengzhaohui@SCMC.com.cn.

Background: Distinguishing biliary atresia from non-biliary atresia in patients with cholestasis is challenging, as these conditions have a similar clinical presentation. We developed and externally validated a screening model for biliary atresia and devised a web-based calculator for use in clinical settings.

Methods: A screening model was developed based on data from 227 cholestatic infants (82 and 145 with and without biliary atresia, respectively) and validated in 234 infants (90 and 144 with and without biliary atresia, respectively) admitted to three hospitals. Variables were selected from routine examination results using the least absolute shrinkage and selection operator method and entered into a logistic regression model to construct a biliary-atresia-risk-predicting equation. Cutoff values for risk stratification were estimated using model sensitivity, derived from the receiver-operating characteristic curves.

Results: The final screening model included seven variables (i.e., weight at admission, clay-colored stools, $\gamma$-glutamyl transpeptidase and albumin levels at admission, abnormal gallbladder, triangular cord sign, and change in total bilirubin levels). The model generated an area under the curve of 0.94 with a sensitivity of 91.46 and specificity of 86.62 in the derivation cohort. This was confirmed in the validation cohort, as we found an area under the curve of 0.93 with a sensitivity of 93.1 and specificity of 80.15 . Patients were stratified into three risk groups (low-, moderate-, and high-risk groups). Biliary atresia was excluded in the low-risk group. The high-risk group showed a higher detection rate of biliary atresia compared to the stool color screening method alone. This model was integrated into a user-friendly web-based system.

Conclusions: The screening tool had a high predictive value and may help in decision-making by physicians at tertiary and community hospitals.

Keywords: Biliary atresia (BA); cholestasis; screening model; infants; liver function tests

Submitted Jul 05, 2020. Accepted for publication Dec 04, 2020.

doi: $10.21037 / \mathrm{tp}-20-170$

View this article at: http://dx.doi.org/10.21037/tp-20-170 


\section{Introduction}

Biliary atresia (BA) is a common cause of cirrhosis and end-stage liver disease in children and, if untreated, can lead to death within the first 2 years of life (1-3). Recently, several studies have provided compelling evidence that early surgical intervention (Kasai portoenterostomy) could significantly improve the prognosis of patients with BA $(4,5)$. When Kasai hepatic portoenterostomy is performed within the first 45 days of life, the survival rates with the native liver can reach $65.5 \%$ and $40.5 \%$ at the age of 2 years and 15 years, respectively (5). Therefore, prompt diagnosis for $\mathrm{BA}$ is required.

Intraoperative cholangiography (IOC) is the gold standard for the diagnosis of BA. It is an invasive procedure that presents a risk of developing adverse effects. Moreover, this surgical procedure requires the skills of experienced surgeons and anesthesiologists, limiting its use in the primary or secondary hospital setting. Lack of interventional exploration could delay diagnosis and treatment; at the same time, referral to a tertiary hospital could expose infants without BA to unnecessary invasive examination. Thus, a screening method is crucial and could lead to significant progress in the timely detection of BA in infants with cholestasis.

Currently, in Japan and Taiwan, screening the pale stools of infants with biliary atresia using the stool color chart has been widely used. This might have resulted in improved treatment times $(6,7)$. However, the sensitivity of stool color card screening at the 1-month check-up was only $76.5 \%$ (7). Besides, the serum direct bilirubin concentration was used for BA screening in an American cohort study and showed high sensitivity and specificity rates (8). However, these screening tools showed an extremely low positive predictive value (5.9\%), which means that elevated direct bilirubin can also been found in conditions with severe intrahepatic cholestasis, such as cytomegalovirus hepatitis $(8,9)$. Jancelewicz et al. developed a screening algorithm for the exclusion of biliary atresia in infants with cholestatic jaundice. However, this algorithm included percutaneous cholangiogram or liver biopsy, which was an invasive procedure (10). This fact limited the potential of these tools as accurate biomarkers for early screening in infants with cholestasis. To date, there is no single non-invasive diagnostic procedure that is clearly superior in the diagnosis of BA (11). Furthermore, infants with BA present with elevated total bilirubin (TBIL), direct bilirubin (DBIL), and liver transaminases levels, including $\gamma$-glutamyl transpeptidase (GGT) levels $(3,12)$. In cases of a progressive congenital cholangiopathy, there are no data supporting the idea that dynamic changes in these variables could help to predict BA.

In this study, we postulated that a multivariate model based on routine noninvasive tests may help screen infants with cholestasis for the presence of BA. Moreover, to facilitate the utilization of our models, we intended to develop an online medical calculator based on the screening model. Thus, our aim was to develop and externally validate a screening model for BA based on routinely available examinations and develop a web-based tool to aid decisionmaking in cases of patient referral and IOC examination. The present manuscript was written in accordance with the TRIPOD reporting checklist (available at http://dx.doi. org/10.21037/tp-20-170) (13).

\section{Methods}

\section{Study design}

This retrospective study was conducted in two-stages and involved three large tertiary hospitals in Shanghai, China. First, to develop a screening model for BA, we obtained a derivation cohort by collecting data from infants with cholestasis admitted to Xinhua Hospital between January 2010 and April 2019. Next, we assembled an external multicenter validation cohort from Shanghai Children's Medical Center (January 2016 to August 2019) and Children's Hospital of Shanghai (June 2014 to August 2019) to validate the performance of this model.

\section{Ethical statement}

The study was conducted in accordance with the Declaration of Helsinki (as revised in 2013). The study was approved by the Institutional Review Board of each participating hospital (ethical approval No. XHEC-C-2019-073) and individual consent for this retrospective analysis was waived.

\section{Participants and data collection}

The inclusion criteria for infants participating in the study were as follows: age at first hospital admission $<100$ days; gestational age $\geq 34$ weeks or birth weight $\geq 2,000 \mathrm{~g}$; DBIL $>20 \%$ of the TBIL for TBIL $>85 \mu \mathrm{mol} / \mathrm{L}$ or DBIL $>17 \mu \mathrm{mol} / \mathrm{L}$ for TBIL $<85 \mu \mathrm{mol} / \mathrm{L}$ (14). At admission, routine investigations were conducted to establish the diagnosis including the liver function test and ultrasound 


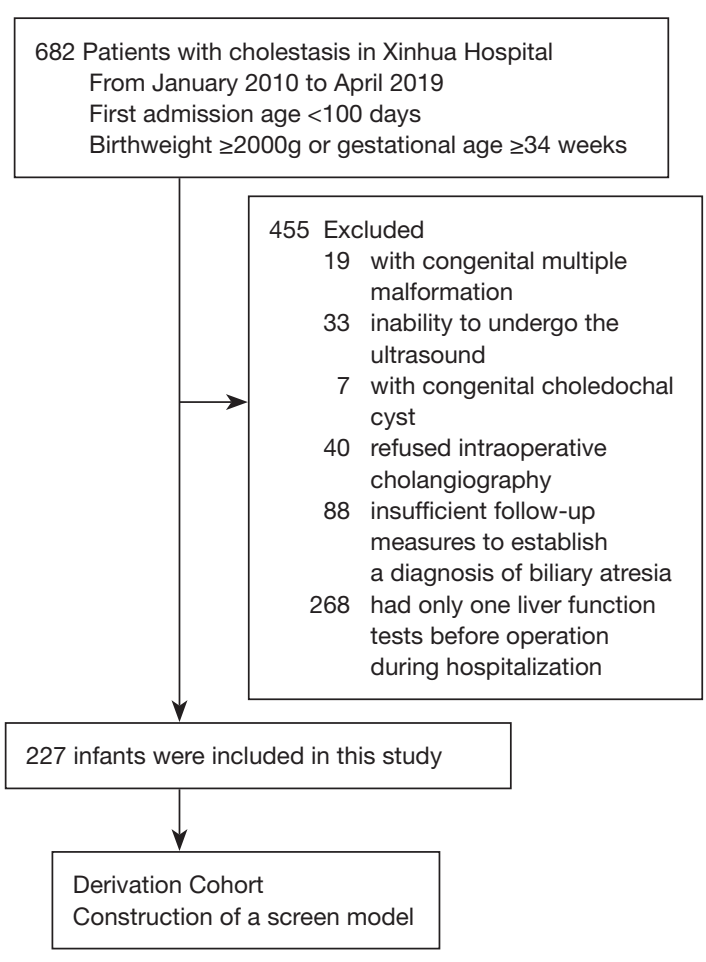

Figure 1 Flowchart of the study population.

(US) examination. The ultimate diagnosis of BA was confirmed by IOC in combination with histological features of intraoperative liver biopsies, which showed abnormal biliary tree, bile duct proliferation, bile plugs, moderate to marked ductular reaction, portal stromal edema, and periportal fibrosis $(3,15,16)$. Non-BA was confirmed by IOC, showing a patent biliary tree or normalized/obviously decreased bilirubin values during hospitalization until discharge or at follow-up.

The exclusion included multiple congenital malformations, congenital choledochal cyst, inability to undergo US scans, refusal to undergo IOC, and insufficient follow-up measurements to establish a diagnosis of biliary atresia. Moreover, to obtain the dynamic change in liver function parameters for each patient, we excluded those who had undergone only one liver function test before operation during hospitalization.

Finally, the data obtained from 227 patients (82 BA and 145 with and without BA, respectively) were used to build a logistic regression model and to determine a screening score. Two hundred and thirty-four patients (90 BA and 144 with and without BA, respectively) were used to build a logistic regression model and to determine

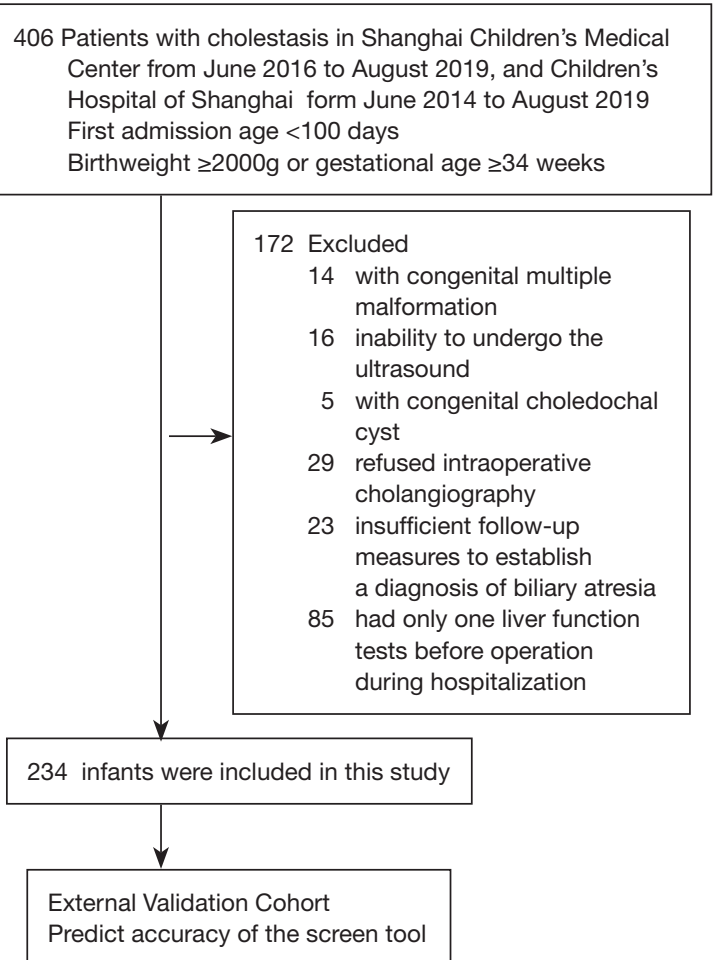

a screening(s) were used to validate the scoring system (Figure 1). Clinical data (i.e., patient demographics; clinical symptoms; parameters of conventional US; and serum TBIL $(\mu \mathrm{mol} / \mathrm{L})$, DBIL $(\mu \mathrm{mol} / \mathrm{L})$, alanine transaminase $(\mathrm{U} / \mathrm{L})$, aspartate transaminase (U/L), alkaline phosphatase (U/L), GGT (U/L), and albumin (g/L) levels were obtained from hospital records. Liver function was assessed on hospital admission and subsequently during routine clinical and laboratory workup at 5-7 days following admission every week until discharge. The dynamic change $(\Delta)$ in liver function parameters was determined by calculating the difference between the value measured at admission and that obtained from the first repeat measurement following admission and before surgical operation. The gallbladder length was assessed by US. Especially, non-visualization of the gallbladder or gallbladder length $\leq 15 \mathrm{~mm}$ was defined as abnormal (17). Triangular cord (TC) sign was defined as the presence of an abnormal triangular or tubular echogenic area in the porta hepatis region (18).

\section{Statistical analyses}

Based on the results of our pilot study, we found that 
Table 1 Demographic characteristics of study population including the derivation and validation data set $(\mathrm{N}=461)$

\begin{tabular}{|c|c|c|c|c|}
\hline Parameters & $\begin{array}{l}\text { Study population } \\
\qquad(\mathrm{n}=461)\end{array}$ & $\begin{array}{l}\text { Derivation data set } \\
\qquad(\mathrm{n}=227)\end{array}$ & $\begin{array}{l}\text { Validation data set } \\
\qquad(\mathrm{n}=234)\end{array}$ & $\begin{array}{l}\text { Validation vs. } \\
\text { derivation, } P\end{array}$ \\
\hline Birth weight, mean (SD), g & $3,157.3 \pm 516.7$ & $3,148.5 \pm 481.8$ & $3,165.9 \pm 549.3$ & 0.719 \\
\hline Age at admission, mean (SD), day & $47.6 \pm 20.7$ & $45.2 \pm 18.4$ & $49.9 \pm 22.5$ & 0.012 \\
\hline Weight at admission mean (SD), g & $4,444.1 \pm 1,082.7$ & $4,242.9 \pm 998.0$ & $4,640.9 \pm 1,127.3$ & $<0.001$ \\
\hline Term, No. (\%) & $414(89.8)$ & $206(90.7)$ & 208 (88.9) & \\
\hline \multicolumn{5}{|l|}{ Sex } \\
\hline Male, No. (\%) & $269(58.4)$ & $137(60.4)$ & $132(56.4)$ & 0.391 \\
\hline
\end{tabular}

$\mathrm{SD}$, standard deviation.

some parameters such as DBIL, GGT, or TC sign were significantly changed in the BA group. The sample size was calculated by these parameters using the SAS power and sample size application 13.2 (SAS Institute Inc., Cary, NC, USA) to calculate $95 \%$ confidence interval (CI) and $80 \%$ power. We calculated and chose the biggest estimated sample size, which was at least 218 participants for the derivation set. Assuming half of the derivation set, we calculated a sample size of at least 109 participants for the external validation set.

In the derivation cohort, we used the least absolute shrinkage and selection operator (LASSO) method to select the potential predictors of BA. LASSO procedures are frequently used in domains with very large datasets, which can shrink small coefficients exactly to zero, resulting in a sparse representation of the solution. Therefore, this procedure could provide a method for variable selection by shrinking the regression coefficients (19). Predictors were identified using the LASSO procedure and inserted into multivariate logistic regression models. The final logistic regression model was fit to identify a screen algorithm for BA. Receiver operating characteristic (ROC) curves were constructed to evaluate the predictive properties of each selected predictor, and the ability of the model to discriminate between patients with and without BA by using the derivation data. This model was applied to the validation cohort to verify diagnostic ability.

To evaluate the effectiveness of the model in risk stratification, we further estimated the risk of BA for participants assigned into three stratified groups: the low-, moderate-, and high-risk groups. The two risk cutoff points that would achieve the desired partitioning were defined as the highest probabilities that maintained sensitivity rates of $100 \%$ and $80 \%$ in the derivation cohort (20). The detection rate in each risk group was calculated for the derivation and validation sets. We also developed a user-friendly online calculator to assist readers with the application of our models.

In univariate analysis, the Chi-squared test was performed for categorical variables, while Student's $t$ test or the Wilcoxon test was used for continuous variables. Descriptive results were expressed as means \pm standard deviations (SD), medians (interquartile ranges, IQRs), or numbers (percentages) of individuals. A statistically significant difference was defined as a $\mathrm{P}$ value $<0.05$. Statistical analysis was performed using SAS 9.2 (SAS Institute, Inc.) and illustrations were plotted using Origin 9 (OriginLab Corp., Northampton, MA, USA).

\section{Results}

\section{Characteristics of derivation and validation cohorts}

The derivation $(\mathrm{n}=227)$ and validation cohorts $(\mathrm{n}=234)$ comprised a total of 461 participants, with 172 (37.3\%) cases of BA observed after performing IOC. The demographic characteristics of the derivation and validation cohorts are presented in Table 1. Overall, 269 male and 192 female infants were enrolled in the study, with a mean admission age of $47.6 \pm 20.7$ days. Compared with the derivation cohorts, patients in the external validation cohort were slightly older and heavier.

The final diagnoses in the non-BA group included 
idiopathic neonatal/infantile hepatitis syndrome $(\mathrm{n}=182)$, cytomegalovirus hepatitis $(\mathrm{n}=81)$, parenteral nutrition associated cholestasis $(n=12)$, Alagille's syndrome $(n=5)$, neonatal intrahepatic cholestasis caused by citrin deficiency $(\mathrm{n}=4)$, sepsis $(\mathrm{n}=2)$, tyrosinemia $(\mathrm{n}=2)$, and Epstein-Barr virus infection $(\mathrm{n}=1)$.

\section{Construction of the BA screen score in the derivation cobort}

In the derivation cohort, we collected basic characteristics to identify cholestatic patients with a risk of BA. There were no significant differences between the BA and non-BA groups in demographic and clinical parameters, including age at admission, sex, recurrent jaundice, hepatomegaly, and splenomegaly $(\mathrm{P}>0.05$, all). Patients with $\mathrm{BA}$ had higher birthweight and weight at admission and higher frequency of clay-colored stools compared with those without BA. Regarding liver function, there were significant differences in the value of $\Delta$ total bile acid, $\Delta$ TBIL, DBIL, $\Delta$ DBIL, and GGT (U/L) at admission between the BA and nonBA groups. The frequency of abnormal gallbladder size and the presence of triangular cord sign were also significantly higher in the BA than in the non-BA group (Table 2).

We applied the LASSO procedure to all variables. Using the LASSO approach, we retained seven measurements, to identify the predictor variables of BA: weight at admission, clay-colored stools, GGT and albumin level at admission, abnormal gallbladder, TC sign, and $\Delta$ TBIL. Figure 2 plots the coefficient progression of the different variables for BA and non-BA in the LASSO model. The seven variables were entered into a logistic regression model to construct an equation predicting the likelihood of BA. Table 3 shows the coefficients obtained from the univariate logistic regression, along with the diagnostic performance of each variable. Significantly elevated odds ratios (ORs) were observed for weight at admission, abnormal gallbladder, triangular cord sign, clay-colored stools, GGT levels at admission, and $\triangle T$ TBIL. A significantly decreased OR was observed for the albumin levels at admission. Among the routine laboratory and US examinations, GGT at admission, clay-colored stools, and triangular cord sign had the highest area under the curve (AUC), highest sensitivity, and highest specificity, respectively.

Finally, we developed the following formula to identify patients with $\mathrm{BA}$ :

$\mathrm{P}=1 /\left(1+1 /\left(\exp \left((-3.5872)+(0.8644)^{*}\right.\right.\right.$ weight at admission + $1.4572^{*}$ clay-colored stools $+1.8654^{*}$ abnormal gallbladder $+0.00317^{\star}$ GGT at admission $+0.0158^{*} \Delta \mathrm{TBIL}+(-0.0807)^{*}$ albumin at admission $+2.5456^{*} \mathrm{TC}$ sign))

The regression coefficients represent per unit change increase in the contribution of each predictor to the calculation of the $\log$ odds of a patient being a case. The overall AUC of the prediction model was 0.94 (95\% CI: 0.899-0.967), with a sensitivity of 91.46 (95\% CI: $83.2-$ $96.5)$ and a specificity of 86.62 (95\% CI: 79.9-91.7). This formula was referred as a screening tool for BA.

To evaluate the effectiveness of the model in risk stratification and clinical utility, we divided patients into three groups (low-, moderate-, and high-risk groups) based on their risk probabilities. The two risk cutoff points were determined to achieve the desired partitioning and were defined as the highest probability maintaining sensitivities of $100 \%$ and $80 \%$, with predicted probabilities of 0.05317 and 0.49998 . As a result, $33.9 \%, 36.6 \%$, and $29.5 \%$ of the total population from the derivation data set were assigned into the high-, intermediate-, and low-risk groups, respectively (Table 4, Figure 3). The detection rate for BA was $85.7 \%$ in the high-risk group. This was higher than the detection rate of the stool color screening method alone and there were no cases of BA in the low-risk group (Table 4).

\section{External validation model}

In the external validation data set, data from 234 patients (90 and 144 with and without BA, respectively) were used to verify the applicability of the BA screen formula. The ROC curve constructed by the screen model yielded an AUC of 0.93 (95\% CI: $0.890-0.962)$ with a sensitivity of 93.1 (95\% CI: 85.6-97.4) and specificity of $80.15(95 \%$ CI: 72.3-86.6), which were similar to those observed in the derivation cohort.

The two cut-off points were applied to the validation date set, and approximately $36.3 \%, 36.3 \%, 27.3 \%$ of patients were classified into the high-, intermediate-, and low-risk groups, respectively. The detection rate for the high-risk group was $84.7 \%$, which was similar to that observed in the derivation cohort and higher than that obtained after using the stool color screening method alone. Interestingly, $80.5 \%$ and $80.0 \%$ of BA cases fell into the high-risk group in the derivation and validation set, respectively, and no case fell into the low-risk group (Table 4, Figure 3). The detection rate for the high-risk group in both datasets was greater than twice compared to the corresponding of the total population (Table 4). 
Table 2 Baseline characteristics of patients with $v s$. without biliary atresia in the derivation cohort $(\mathrm{N}=227)$

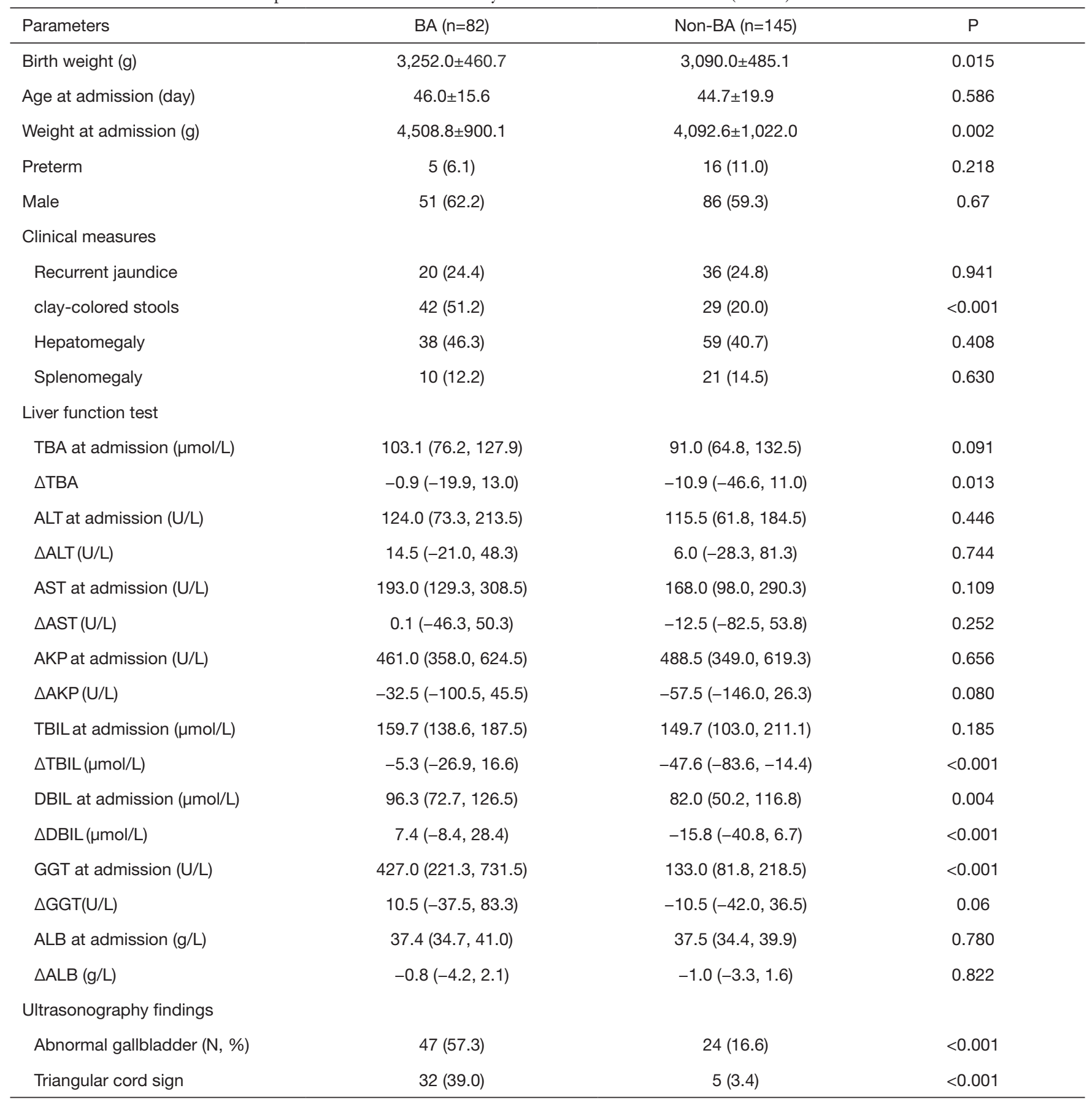

BA, biliary atresia; TBA, total bile acid; ALT, alanine transaminase; AST, aspartate transaminase; AKP, alkaline phosphatase; TBIL, total bilirubin; DBIL, direct bilirubin; GGT, $\gamma$-glutamyl transferase; ALB, albumin. Data are presented as mean \pm standard deviation, $\mathrm{N}(\%)$, or median (IQR, inter quartile range). 


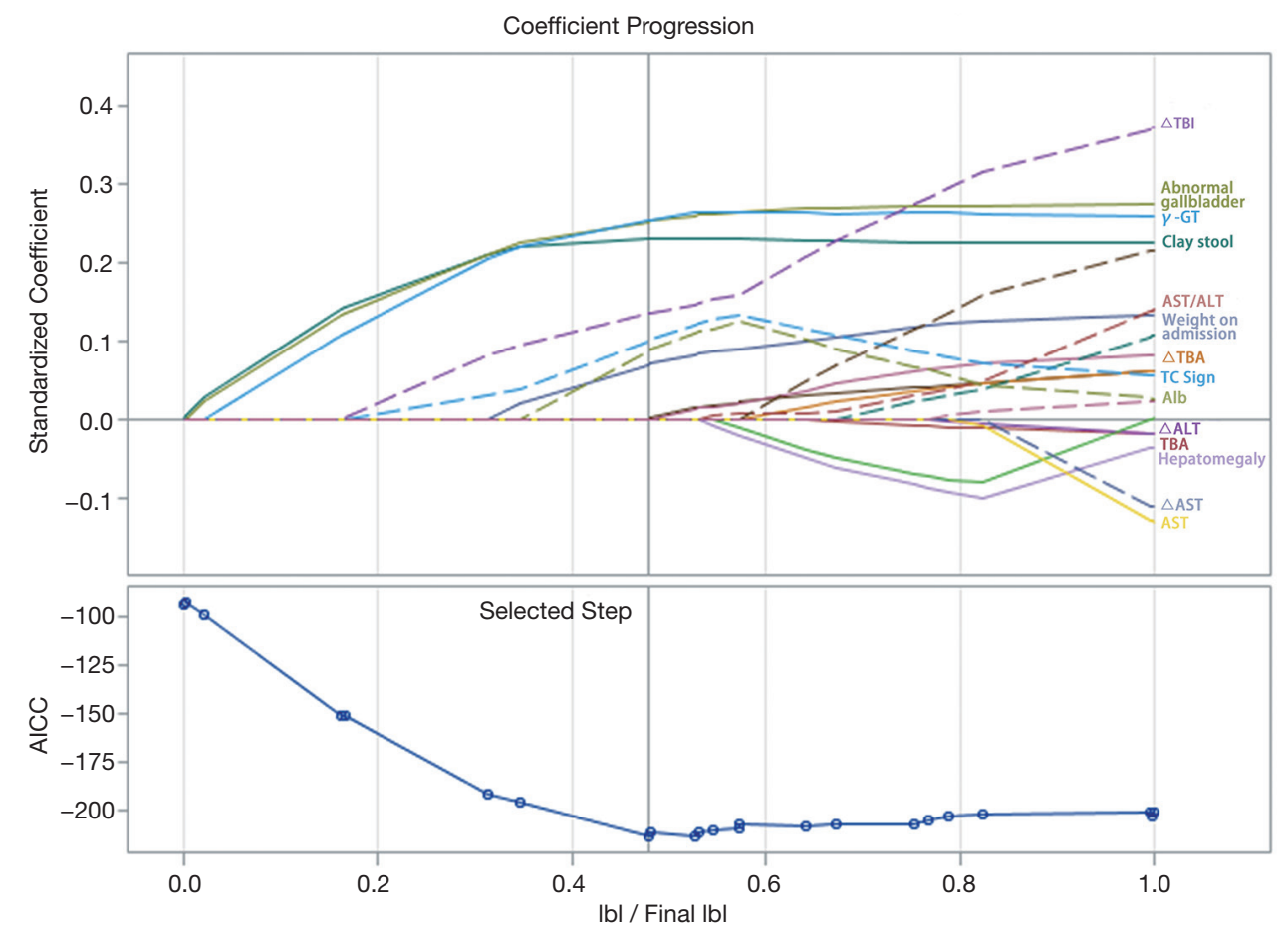

Figure 2 Selection of biliary atresia associated predictors by using the LASSO logistic regression algorithm. The top figure shows the LASSO selection process. Standardized coefficients of all the effects selected at some point of the stepwise method are plotted as a function of the step number. The vertical line corresponds to the model that minimizes AICC. The bottom figures show AICC values for candidate models along solution path. The vertical line corresponds to the model with seven variables and smallest AICC value. Seven predictors were selected from the model, including weight at admission, abnormal gallbladder, triangular cord sign, clay-colored stools, GGT at admission, albumin at admission, and $\Delta$ Total bilirubin. LASSO, least absolute shrinkage and selection operator; AICC, corrected Akaike's information criterion; GGT, $\gamma$-glutamyl transpeptidase.

\section{Clinical usefulness of the risk calculator}

To facilitate the utilization of our model, we developed a detailed user-friendly online version of the risk calculator (available free of charge at http://infantsmc.cn/exp/). This was developed to allow clinicians to use the screening tool for rapid calculation of BA risk in patients, facilitating the decision making in clinical practice.

An example of risk calculation is presented: in the validation cohort, a 50-day-old infant with cholestasis underwent routine investigations and repeated liver function tests at 5 days after admission. The following data obtained at admission were entered: weight, $4.5 \mathrm{~kg}$; GGT, $585 \mathrm{U} / \mathrm{L}$; albumin, $33.9 \mathrm{~g} / \mathrm{L}$, TBIL, $126.7 \mu \mathrm{mol} / \mathrm{L}$; repeated TBIL, $141.2 \mu \mathrm{mol} / \mathrm{L}$; presence of the clay-colored stool; presence of an abnormal gallbladder; and positive TC sign. Using the calculator, we obtained an overall predictive ability of 0.99993. The infant was classified into the high-risk group and the IOC was recommended by the tool.

\section{Discussion}

In this study, we proposed a BA screening tool using routine demographic, clinical, laboratory, and radiological variables to discriminate between $\mathrm{BA}$ and other cholestatic disorders. By combining seven easily obtained predictors (including weight at admission, the stool color card value, GGT and albumin at admission, presence of an abnormal gallbladder, TC sign, and $\triangle$ TBIL), our final model achieved high discrimination and capacity with an AUC of 0.94 , sensitivity of 91.46 , and specificity of 86.62 in the derivation cohort, and an AUC of 0.93 , sensitivity of 93.1, and specificity of 80.15 in an externally validated cohort. Overall, $80.5 \%$ and $80 \%$ of BA cases fell in the high-risk group in the derivation and validation sets, respectively, and no case fell in the low-risk group. The detection rate in the 
Table 3 Univariate logistic regression analysis and diagnostic performance of selected parameters associated with biliary atresia in the derivation cohort

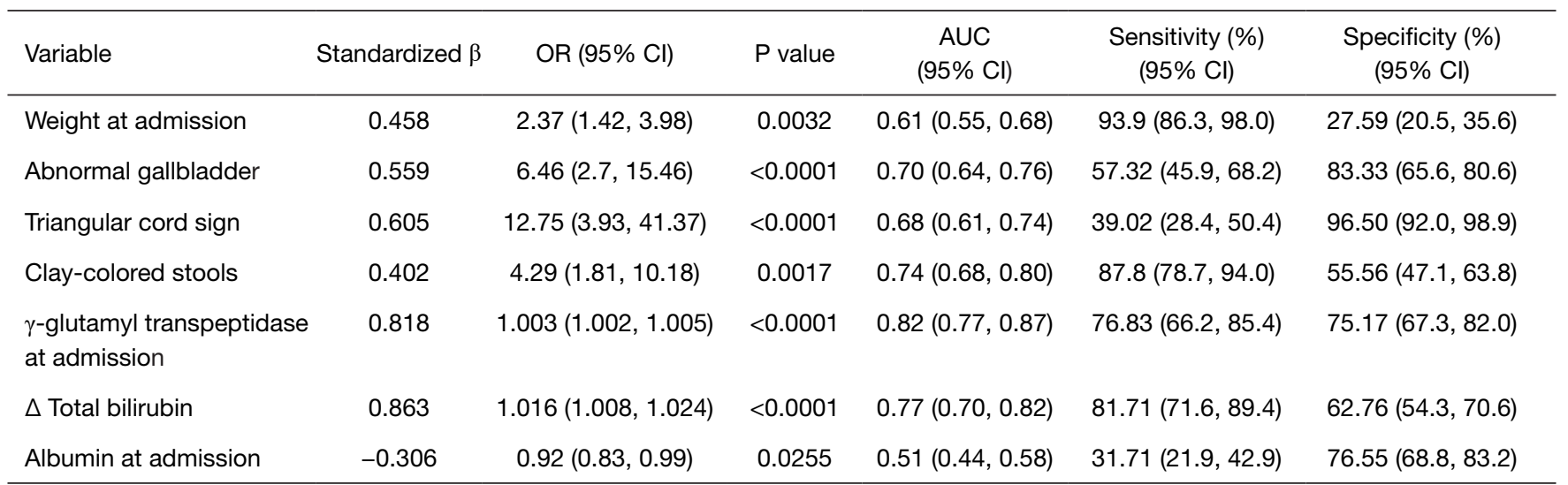

AUC, area under the curve; $\mathrm{Cl}$, confidence interval.

Table 4 Effectiveness of risk stratification based on the screen model and clay stool of biliary atresia in derivation and validation cohort

\begin{tabular}{|c|c|c|c|c|c|c|c|c|c|}
\hline $\begin{array}{l}\text { Biliary atresia } \\
\text { screen method }\end{array}$ & Cutoffs & \multicolumn{4}{|c|}{ Derivation cohort $(n=227)$} & \multicolumn{4}{|c|}{ Validation cohort $(n=234)$} \\
\hline $\begin{array}{l}\text { High risk group } \\
\text { in the screen } \\
\text { model }\end{array}$ & $\geq 0.49998$ & 77 (33.9) & $66(80.5)$ & 85.7 & $\begin{array}{c}82 / 227 \\
(36.1)\end{array}$ & $85(36.3)$ & $72(80.0)$ & 84.7 & $\begin{array}{c}90 / 234 \\
(38.5)\end{array}$ \\
\hline $\begin{array}{l}\text { Intermediate } \\
\text { risk group in the } \\
\text { screen model }\end{array}$ & $\begin{array}{l}<0.49998 \\
>0.05317\end{array}$ & $83(36.6)$ & $16(19.5)$ & 19.3 & & $85(36.3)$ & $18(20.0)$ & 21.2 & \\
\hline $\begin{array}{l}\text { Clay-colored } \\
\text { stools in the } \\
\text { total population }\end{array}$ & Presence & $71(31.3)$ & $42(51.2)$ & 59.2 & & $62(26.5)$ & $35(38.9)$ & 56.5 & \\
\hline
\end{tabular}

high-risk group was much higher than that obtained using the stool color method alone. Moreover, in our study, a userfriendly online version of the risk calculator demonstrated satisfactory outcomes, allowing the widespread use of this model in clinical practice. Our tool may be valuable in making decisions to screen patients and highlights the importance of avoiding invasive procedures in low-risk patients, thus, limiting the performance of IOC to high-risk patients.

To explore a combined screening tool for the diagnosis of BA using basic investigations that could be performed routinely, we enrolled three tertiary care hospitals in Shanghai receiving referrals of cases of cholestatic infants from across China. In our prediction model, the estimated risk may help distinguish individuals with different levels of risk. We recommended IOC for individuals at high risk. Surgical exploration was not recommended for patients with a low BA risk. Further investigations, such as hepatobiliary scintigraphy and liver biopsy, were recommended in 
A Probability of biliary atresia by disease in the derivation cohort

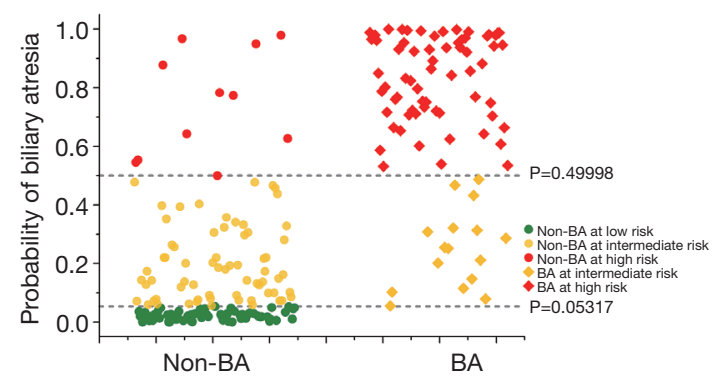

B Probability of biliary atresia by disease in the validation cohort

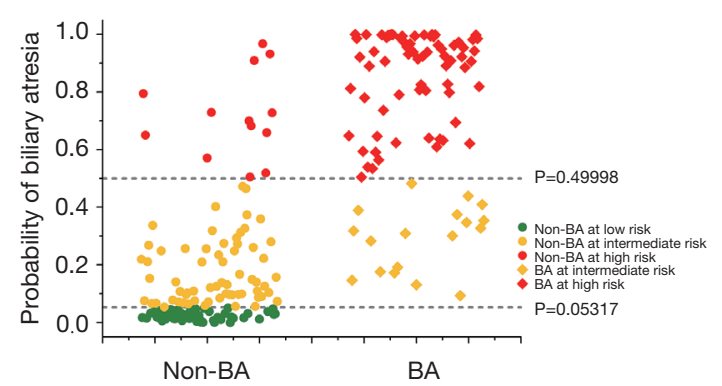

Figure 3 Biliary atresia screening model in individual infants in the derivation cohort and the validation cohort. This model had an AUC of 0.94 (95\% CI: 0.899-0.967) in the derivation cohort and an AUC of 0.93 (95\% CI: 0.890-0.962) in the validation cohort. The dashed line represents the two cutoff values (0.05317 and 0.49998 ) for the high-, intermediate-, and low group. In the highrisk group, the detection rate of BA was $85.7 \%$ in the derivation cohort and $84.7 \%$ in the validation cohort, and no case fell in the low-risk group in the derivation cohort (a) and in the validation cohort (b), respectively. AUC, area under the curve; CI, confidence interval; BA, biliary atresia.

patients with an intermediate risk of BA.

Seven final predictors were identified through the LASSO method. Unlike standard multiple linear regression, LASSO can control multicollinearity resulting from highly correlated variables and performs shrinkage and automatic variable selection simultaneously (21). Of these, claycolored stools had the highest sensitivity, which was one key feature suggestive of BA. In some regions, stool color cards have been used for mass screening for BA with high sensitivity $(7,22)$. Our results showed that in cholestatic patients, clay-colored stools had a sensitivity of $87.8 \%$, specificity of $55.56 \%$, and the detection rate of BA was much lower than that in the high-risk group in both cohorts. US, another simple and noninvasive procedure, was widely used. Several US findings have been described as useful indicators of BA. Especially, the gallbladder length and triangular cord sign, easily measured by conventional US, are useful in the diagnosis of BA (23). Our study found that TC sign had the highest specificity $(96.50 \%)$, which was consistent with previous studies (24). However, its poor sensitivity (39.02\%), indicated that diagnosis of BA should not solely rely on this method.

Routinely-available liver function tests, which are useful in the identification of BA, were also included in this model. The usefulness of GGT to identify BA has been extensively investigated and demonstrated $(3,11)$. Interestingly, we found that $\triangle \mathrm{TBIL}$ concentration was higher in the BA than in the non-BA group $(\mathrm{P}<0.001)$. $\Delta$ TBIL also had a high standardized $\beta$ value in the model, indicating that it had a greater effect on the screening tool. Previous studies have reported that elevated conjugated bilirubin levels could be used to screen for BA in neonates (25). In our study, DBIL was not included in this model using the LASSO method. However, we found that elevated or slightly decreased TBIL levels had a sensitivity of $81.71 \%$ for the diagnosis of BA in infants with cholestasis. Observing the dynamic changes in TBIL that might be more significant than TBIL levels in identifying BA, since BA is a progressive hepatobiliary disorder in infants with cholestasis. The Kasai procedure is recommended within the first 60 days of life in infants with BA, to achieve significant bile drainage (26). Therefore, repeated liver function tests and observation procedures within 1 week in cholestatic infants aged $<60$ days are recommended, as this method does not delay diagnosis and avoids unnecessary surgical intervention.

Recent studies have investigated the use of other scoring systems to identify BA in infants with cholestasis $(3,11,17,27)$. Kim et al. reported that accurate diagnosis of BA among neonates with cholestasis was achieved using a new scoring system that combined clinical, US, and hepatobiliary scintigraphy findings (17). El-Guindi et al. devised an accurate diagnostic scoring system with high sensitivity and specificity based on the histopathological evaluation of liver biopsy, which is an invasive procedure (11).

Although these scoring systems may have greater accuracy, our study, which was developed and validated in a large cohort of patients with BA, presented several strengths. First, the clinical and laboratory parameters used in our screening tool were non-invasive, readily available, and routinely ordered during hospital admission, even in the primary and secondary hospital setting. Furthermore, the final optimized BA screening tool is convenient to use with a freely accessible 
website that enables the clinicians to calculate the BA risk and, therefore, may aid in decision making regarding the appropriate patient care. Finally, we set up two cutoff points to classify patients into three risk stratification groups. Approximately $30 \%$ of patients were classified as having low risk, and none of these patients were diagnosed with BA, indicating that this screening tool has an excellent predictive capability for excluding BA in the derivation and validation cohorts. Therefore, particularly in the primary hospital setting, patients could avoid undergoing unnecessary invasive procedures, with savings in respect of the costs of referral to and medical expenses incurred in large tertiary care hospitals.

However, some limitations of this study should be addressed. First, the study protocol required all enrolled patients to undergo two sets of blood tests to observe changes in liver function. Thus, the number of enrolled patients was lower than overall number of patients who underwent IOC but were excluded, as they did not have undergone two liver function tests. Second, this screening tool did not include more accurate examinations, such as hepatobiliary scintigraphy and liver biopsy (28), that could be routinely requested in a tertiary referral center. Nevertheless, the BA screening tool allows clinicians to use commonly available laboratory parameters to make an initial assessment, which would trigger further investigations.

\section{Conclusions}

In summary, our study provided a web-based clinical tool for BA screening in clinical practice with low-cost and easy-to-use stratification. This tool presented a high accuracy of prediction and its performance was validated in an independent population. Using this tool, clinicians can identify BA in patients at an early stage of disease and proceed promptly with IOC and Kasai portoenterostomy in the high-risk group. The tool allows patients with a low risk of BA to be discharged without the need for invasive exploration. The web-based tool could help health-care providers estimate stratification of the individualized BA risk by inputting routinely available laboratory and demographic-based variables. Therefore, its use would be of great importance, as the physicians working at the tertiary and community hospitals would easily identify cases of BA.

\section{Acknowledgments}

We acknowledge https://app.editage.com/ for English checking.
Funding: None.

\section{Footnote}

Reporting Checklist: The authors have completed the TRIPOD reporting checklist. Available at http://dx.doi. org/10.21037/tp-20-170

Data Sharing Statement: Available at http://dx.doi. org/10.21037/tp-20-170

Conflicts of Interest: All authors have completed the ICMJE uniform disclosure form (available at http://dx.doi. org/10.21037/tp-20-170). The authors have no conflicts of interest to declare.

Ethical Statement: The authors are accountable for all aspects of the work in ensuring that questions related to the accuracy or integrity of any part of the work are appropriately investigated and resolved. The study was conducted in accordance with the Declaration of Helsinki (as revised in 2013). The study was approved by the Institutional Review Board of each participating hospital (ethical approval No. XHEC-C-2019-073) and individual consent for this retrospective analysis was waived.

Open Access Statement: This is an Open Access article distributed in accordance with the Creative Commons Attribution-NonCommercial-NoDerivs 4.0 International License (CC BY-NC-ND 4.0), which permits the noncommercial replication and distribution of the article with the strict proviso that no changes or edits are made and the original work is properly cited (including links to both the formal publication through the relevant DOI and the license). See: https://creativecommons.org/licenses/by-nc-nd/4.0/.

\section{References}

1. Moreira RK, Cabral R, Cowles RA, et al. Biliary atresia: a multidisciplinary approach to diagnosis and management. Arch Pathol Lab Med 2012;136:746-60.

2. Bezerra JA, Wells RG, Mack CL, et al. BILIARY ATRESIA: Clinical and Research Challenges for the 21(st) Century. Hepatology 2018;68:1163-73.

3. Dong R, Jiang J, Zhang S, et al. Development and Validation of Novel Diagnostic Models for Biliary Atresia in a Large Cohort of Chinese Patients. EBioMedicine 2018;34:223-30. 
4. Davenport M, Caponcelli E, Livesey E, et al. Surgical outcome in biliary atresia: etiology affects the influence of age at surgery. Ann Surg 2008;247:694-8.

5. Serinet MO, Wildhaber BE, Broue P, et al. Impact of age at Kasai operation on its results in late childhood and adolescence: a rational basis for biliary atresia screening. Pediatrics 2009;123:1280-6.

6. Lin JS, Chen SC, Lu CL, et al. Reduction of the ages at diagnosis and operation of biliary atresia in Taiwan: A 15year population-based cohort study. World J Gastroenterol 2015;21:13080-6.

7. Gu YH, Yokoyama K, Mizuta K, et al. Stool color card screening for early detection of biliary atresia and longterm native liver survival: a 19-year cohort study in Japan. J Pediatr 2015;166:897-902.e1.

8. Harpavat S, Garcia-Prats JA, Anaya C, et al. Diagnostic Yield of Newborn Screening for Biliary Atresia Using Direct or Conjugated Bilirubin Measurements. JAMA 2020;323:1141-50.

9. Oliveira NL, Kanawaty FR, Costa SC, et al. Infection by cytomegalovirus in patients with neonatal cholestasis. Arq Gastroenterol 2002;39:132-6.

10. Jancelewicz T, Barmherzig R, Chung CT, et al. A screening algorithm for the efficient exclusion of biliary atresia in infants with cholestatic jaundice. J Pediatr Surg 2015;50:363-70.

11. El-Guindi MA, Sira MM, Sira AM, et al. Design and validation of a diagnostic score for biliary atresia. J Hepatol 2014;61:116-23.

12. Harpavat S, Finegold MJ, Karpen SJ. Patients with biliary atresia have elevated direct/conjugated bilirubin levels shortly after birth. Pediatrics 2011;128:e1428-33.

13. Bossuyt PM, Reitsma JB, Bruns DE, et al. STARD 2015: An Updated List of Essential Items for Reporting Diagnostic Accuracy Studies. Clin Chem 2015;61:1446-52.

14. Fawaz R, Baumann U, Ekong U, et al. Guideline for the Evaluation of Cholestatic Jaundice in Infants: Joint Recommendations of the North American Society for Pediatric Gastroenterology, Hepatology, and Nutrition and the European Society for Pediatric Gastroenterology, Hepatology, and Nutrition. J Pediatr Gastroenterol Nutr 2017;64:154-68.

15. Hartley J, Harnden A, Kelly D. Biliary atresia. BMJ 2010;340:c2383.

16. Russo P, Magee JC, Anders RA, et al. Key Histopathologic Features of Liver Biopsies That Distinguish Biliary Atresia From Other Causes of Infantile Cholestasis and Their Correlation With Outcome: A Multicenter Study. Am J
Surg Pathol 2016;40:1601-15.

17. Kim JR, Hwang JY, Yoon HM, et al. Risk Estimation for Biliary Atresia in Patients with Neonatal Cholestasis: Development and Validation of a Risk Score. Radiology 2018;288:262-9.

18. Lee HJ, Lee SM, Park WH, et al. Objective criteria of triangular cord sign in biliary atresia on US scans. Radiology 2003;229:395-400.

19. Zhao YJ, Zhang HJ, Li CX, et al. Selecting placental measures that have clinical implications in child development and diseases. Placenta 2014;35:178-87.

20. Liu Z, Guo C, He Y, et al. A clinical model predicting the risk of esophageal high-grade lesions in opportunistic screening: a multicenter real-world study in China. Gastrointest Endosc 2020;91:1253-60.e3.

21. Chen Y, Zou L, Zhao Y, et al. Creating a placental inflammatory composite index that has a high prognostic relevance to child morbidity. J Obstet Gynaecol Res 2017;43:1169-79.

22. Tseng JJ, Lai MS, Lin MC, et al. Stool color card screening for biliary atresia. Pediatrics 2011;128:e1209-15.

23. Tan Kendrick AP, Phua KB, Ooi BC, et al. Making the diagnosis of biliary atresia using the triangular cord sign and gallbladder length. Pediatr Radiol 2000;30:69-73.

24. Kanegawa K, Akasaka Y, Kitamura E, et al. Sonographic diagnosis of biliary atresia in pediatric patients using the "triangular cord" sign versus gallbladder length and contraction. AJR Am J Roentgenol 2003;181:1387-90.

25. Harpavat S, Garcia-Prats JA, Shneider BL. Newborn Bilirubin Screening for Biliary Atresia. N Engl J Med 2016;375:605-6.

26. Wong KK, Chung PH, Chan IH, et al. Performing Kasai portoenterostomy beyond 60 days of life is not necessarily associated with a worse outcome. J Pediatr Gastroenterol Nutr 2010;51:631-4.

27. Liu X, Peng X, Huang Y, et al. Design and validation of a noninvasive diagnostic criteria for biliary atresia in infants based on the STROBE compliant. Medicine (Baltimore) 2019;98:e13837.

28. Nakamura H, Yamataka A. Non-invasive and accurate diagnostic system for biliary atresia. EBioMedicine 2018;36:16-7.

Cite this article as: Zhao D, Gu S, Gong X, Li Y, Sun X, Chen Y, Deng Z, Zhang Y. Web-based calculator for biliary atresia screening in neonates and infants with cholestasis. Transl Pediatr 2021;10(2):225-235. doi: 10.21037/tp-20-170 